\title{
Large $N_{c} \mathbf{Q C D}$ and quark model for charm and bottom baryons
}

\author{
Fabien Buisseret ${ }^{*}$ \\ University of Mons-Hainaut, Place du Parc 20, B-7000 Mons, Belgium \\ E-mail: fabien.buisseret@umh.ac.be
}

\section{Claude Semay}

University of Mons-Hainaut, Place du Parc 20, B-7000 Mons, Belgium

E-mail: claude.semay@umh.ac.be

\section{Florica Stancu}

University of Liège, Insitute of Physics B5, Sart Tilman, B-4000 Liège, Belgium

E-mail: fstancululg.ac.be

\section{Nicolas Matagne}

Institut für Theoretische Physik, Universität Giessen, D-35392 Giessen, Germany

E-mail: Nicolas.Matagnedtheo.physik.uni-giessen.de

\begin{abstract}
We establish a connection between the quark model and the $1 / N_{c}$ expansion mass formulas used in the description of baryon resonances. First, we show that a remarkable compatibility exists between the two methods in the light baryon sector. Second, we extend our study to the baryons made of one heavy quark and two light quarks. It appears that the band number used to classify baryons in large $N_{c}$ QCD is explained by the quark model, and the mass formulas for both approaches are consistent. In particular, the quark model reproduces the coefficients of the $1 / N_{c}$ mass formula for the ground state charm and bottom baryons with all the parameters fitted to light baryons.
\end{abstract}

8th Conference Quark Confinement and the Hadron Spectrum

September 1-6, 2008

Mainz. Germany

\footnotetext{
* Speaker.

$\dagger$ F.R.S.-FNRS Postdoctoral Researcher

${ }^{\ddagger}$ F.R.S.-FNRS Senior Research Associate
} 


\section{Baryons in large $N_{c}$ QCD}

\subsection{Light baryons}

In large $N_{c} \mathrm{QCD}$, the gauge group is $\mathrm{SU}\left(N_{c}\right)$ and a baryon is a bound state of $N_{c}$ quarks. The $1 / N_{c}$ expansion is based on the discovery that, in the limit $N_{c} \rightarrow \infty$, QCD possesses an exact contracted $\mathrm{SU}\left(2 N_{f}\right)$ symmetry where $N_{f}$ is the number of flavors. This symmetry is approximate for finite $N_{c}$ so that corrections have to be added in powers of $1 / N_{c}$. When $\mathrm{SU}\left(N_{f}\right)$ is exact the mass operator $M$ has the general form

$$
M=\sum_{i} c_{i} O_{i}
$$

where the coefficients $c_{i}$ encode the QCD dynamics and have to be determined from a fit to the existing data, and where the operators $O_{i}$ are $\mathrm{SU}\left(2 N_{f}\right) \otimes \mathrm{SO}(3)$ scalars of the form

$$
O_{i}=\frac{1}{N_{c}^{n-1}} O_{\ell}^{(k)} \cdot O_{S F}^{(k)}
$$

Here $O_{\ell}^{(k)}$ is a $k$-rank tensor in $\mathrm{SO}(3)$ and $O_{S F}^{(k)}$ a $k$-rank tensor in $\mathrm{SU}(2)$-spin, but invariant in $\mathrm{SU}\left(N_{f}\right)$-flavor. $n$ represents the minimum of gluon exchanges to generate the operator. In practical applications, it is customary to include terms up to $1 / N_{c}$ and drop higher order corrections of order $1 / N_{c}^{2}$. The classification scheme used in the $1 / N_{c}$ expansion for baryon resonances is based on the standard SU(6) classification as in a constituent quark model. Baryons are grouped into excitation bands $N=0,1,2, \ldots$, each band containing at least one $\mathrm{SU}(6)$ multiplet, the band number $N$ being the total number of excitation quanta in a harmonic oscillator picture. Note that the coefficients $c_{i}$ depend on $N$.

One obviously has to set $N_{f}=2$ for light nonstrange baryons. As an example, the ground state mass formula reads in this case $M=c_{1} N_{c} \mathbf{1}+c_{4} S^{2} / N_{c}+\mathscr{O}\left(N_{c}^{-3}\right)$. Other terms like spin-orbit and isospin-dependent contributions [1] appear in excited bands. For light strange baryons, $N_{f}=3$ and one has to add a general mass term of the form

$$
n_{s} \Delta M_{s}=\sum_{i=1} d_{i} B_{i}
$$

where $n_{s}$ is the number of strange quarks and where the operators $B_{i}$ break $\mathrm{SU}(3)$-flavor symmetry.

\subsection{Heavy quarks}

The approximate spin-flavor symmetry for large $N_{c}$ baryons containing two light $q=\{u, d, s\}$ and one heavy $Q=\{c, b\}$ quark is $\mathrm{SU}(6) \times \mathrm{SU}(2)_{c} \times \mathrm{SU}(2)_{b}$, i.e. there is a separate spin symmetry for each heavy flavor. For these baryons, a $1 / m_{Q}$ expansion can be combined to the $1 / N_{c}$ expansion, $m_{Q}$ being the heavy quark mass. If the $\mathrm{SU}(3)$-flavor symmetry is exact, the mass operator reads

$$
M=m_{Q} \mathbf{1}+c_{0} N_{c} \mathbf{1}+\frac{c_{2}}{N_{c}} J_{q q}^{2}+\frac{c_{0}^{\prime}}{2 m_{Q}} \mathbf{1}+\frac{c_{2}^{\prime}}{2 m_{Q} N_{c}^{2}} J_{q q}^{2}+2 \frac{c_{2}^{\prime \prime}}{N_{c} m_{Q}} \vec{J}_{q q} \cdot \vec{J}_{Q}
$$

where $\vec{J}_{q q}\left(\vec{J}_{Q}\right)$ is identical to the total spin $\vec{S}_{q q}\left(\vec{S}_{Q}\right)$ of the light quark pair (of the heavy quark) when one deals with the $N=0$ band. The unknown coefficients have to be fitted to experimental 
data. Physical and dimensional arguments suggest to introduce a typical QCD energy scale $\Lambda$ and to set

$$
c_{0}=\Lambda, \quad c_{2} \sim \Lambda, \quad c_{0}^{\prime} \sim c_{2}^{\prime} \sim c_{2}^{\prime \prime} \sim \Lambda^{2} .
$$

The inclusion of SU(3)-flavor breaking leads to an expansion of the mass operator in the SU(3)-violating parameter $\varepsilon \sim\left(m_{s}-m\right) \sim 0.2-0.3$, where $m$ is the average mass of the $u, d$ quarks and where $m_{s}$ is the strange quark mass. Its value is measured in units of the chiral symmetry breaking scale parameter $\Lambda_{\chi} \sim 1 \mathrm{GeV}$.

\section{Quark model}

A baryon, viewed as a bound state of three quarks, can be described in a first approximation by the spinless Salpeter Hamiltonian $H=\sum_{i=1}^{3} \sqrt{\vec{p}_{i}^{2}+m_{i}^{2}}+\sigma \sum_{i=1}^{3}\left|\vec{x}_{i}-\vec{R}\right|$, where $m_{i}$ is the current quark mass and where $\sigma$ is the string tension. The confinement is given by a Y-junction in which the Toricelli point is replaced by $\vec{R}$, the position of the center of mass. $k$ is a corrective factor, equal to $k_{0}=0.952\left(k_{1}=0.930\right)$ for $q q q(q q Q)$ baryons. It is also necessary to include some perturbative corrections, namely one-gluon exchange and quark self-energy mass terms, respectively reading

$$
\Delta M_{\mathrm{oge}}=-\frac{2}{3} \sum_{i<j=1}^{3}\left\langle\frac{\alpha_{s, i j}}{\left|\vec{x}_{i}-\vec{x}_{j}\right|}\right\rangle, \quad \Delta M_{\mathrm{qse}}=-\frac{f a}{2 \pi} \sum_{i=1}^{3} \frac{\eta\left(m_{i} / \delta\right)}{\mu_{i}} .
$$

$\alpha_{s, i j}$ is the strong coupling constant between the quarks $i$ and $j$ and $\mu_{i}=\left\langle\sqrt{\vec{p}_{i}^{2}+m_{i}^{2}}\right\rangle$ is the kinetic energy of the quark $i$. The factors $3 \leq f \leq 4$ and $(1.0 \leq \delta \leq 1.3) \mathrm{GeV}$ have been computed in lattice QCD. $\eta(x)$ is analytically known and can accurately be fitted by $\eta(x) \approx 1-\beta x^{2}$ with $\beta=2.85$ for $0 \leq x \leq 0.3$ and by $\gamma / x^{2}$ with $\gamma=0.79$ for $1.0 \leq x \leq 6.0$.

Within our model, we have $m_{u}=m_{d}=0$. In this case, using the auxiliary field technique, analytical mass formulas can be obtained for both light $q q q$ and heavy $q q Q$ baryons at the order $\mathscr{O}\left(m_{s}^{2}\right)$ and $\mathscr{O}\left(1 / m_{Q}\right)$. For light baryons one has $M_{q q q}=M_{0}+n_{s} \Delta M_{0 s}\left(n_{s}=0,1,2,3\right)$ with $[2,3]$

$$
M_{0}=6 \mu_{0}-\frac{2 \pi \sigma \alpha_{0}}{6 \sqrt{3} \mu_{0}}-\frac{f \sigma}{4 \mu_{0} k_{0}}, \quad \Delta M_{0 s}=\frac{m_{s}^{2}}{\mu_{0}}\left[\frac{1}{2}-\frac{\pi \sigma \alpha_{0}}{36 \sqrt{3} \mu_{0}^{2}}+\frac{f \sigma}{12 \pi k_{0}}\left(\frac{3}{4 \mu_{0}^{2}}+\frac{\beta}{\delta^{2}}\right)\right] .
$$

In these equations, $\mu_{0}=\sqrt{\pi \sigma(N+3) / 18}$, and $\alpha_{0}=\alpha_{s, q q}$. Moreover, $N$ is the baryon band number in a harmonic oscillator picture, just as the one which is used in large $N_{c}$ QCD. This allows a direct comparison between both approaches.

For heavy baryons one has $M_{q q Q}=m_{Q}+M_{1}+n_{s} \Delta M_{1 s}+\Delta M_{Q}\left(n_{s}=0,1,2\right)$, with [4]

$$
\begin{aligned}
M_{1} & =4 \mu_{1}-\frac{2}{3}\left(\alpha_{0} \sqrt{\frac{k_{1} \pi \sigma}{18 k_{0}}}+2 \alpha_{1} \sqrt{\frac{k_{1} \pi \sigma}{3 k_{0}(N+3)}}\right)-\frac{f \sigma}{6 k_{0} \mu_{1}} \\
\Delta M_{Q} & \left.=\frac{k_{1} \pi \sigma}{12 k_{0} m_{Q}}\left[\left(1-\frac{f \sigma}{12 k_{0} \mu_{1}^{2}}\right) F(N)-\frac{\alpha_{0}}{6} \sqrt{\frac{2 N+3}{3}}\left(\sqrt{\frac{2(2 N+3)}{N+3}}-1\right)+\frac{4 \alpha_{1}}{3} \frac{2 N+3}{N+3}\right]\right] \\
\Delta M_{1 s} & =\frac{m_{s}^{2}}{\mu_{1}}\left[\frac{1}{2}-\frac{1}{12 \mu_{1}}\left(\alpha_{0} \sqrt{\frac{k_{1} \pi \sigma}{18 k_{0}}}+2 \alpha_{1} \sqrt{\frac{k_{1} \pi \sigma}{3 k_{0}(N+3)}}\right)+\frac{f \sigma}{12 k_{0}}\left(\frac{3}{4 \mu_{1}^{2}}+\frac{\beta}{\delta^{2}}\right)\right.
\end{aligned}
$$


Moreover, $\mu_{1}=\sqrt{k_{1} \pi \sigma(N+3) / 12 k_{0}}, F(N)=\sqrt{2 N+3}(\sqrt{2(N+3)}-\sqrt{2 N+3})$, and $\alpha_{1}=\alpha_{s, q Q}=$ $0.7 \alpha_{0}$. The band number $N$ corresponds this time to the relative quantum of excitation of the heavy quark and the light quark pair. The heavy quark-light diquark picture is favored since the quark pair tends to remain in its ground state [4].

\section{Comparison of both approaches}

\subsection{Light baryons}

The coefficients $c_{i}$ appearing in the $1 / N_{c}$ mass operator can be obtained from a fit to experimental data and compared with the quark model results. The dominant term $c_{1} N_{c}$ in the mass formula (1.1) contains the spin- and strangeness-independent mass contributions, which in a quark model language represents the confinement and the kinetic energy. So, for $N_{c}=3$, we expect

$$
c_{1}^{2}=M_{0}^{2} / 9 .
$$

Figure 1 shows a comparison between the values of $c_{1}^{2}$ obtained in the $1 / N_{c}$ expansion method and those derived from Eq. (3.1) for various values of $N$. One can see that the results of large $N_{c}$ QCD are entirely compatible with the formula (3.1) for standard values of the parameters. The spin-dependent corrections between quarks $i$ and $j$ should be of order $O\left(1 / \mu_{i} \mu_{j}\right)$. Therefore we expect both $c_{2}$ and $c_{4}$ to be proportional to $(N+3)^{-1}$ : Such a behavior is consistent with the large $N_{c}$ results, where it is also observed that the spin-spin contribution $\left(c_{4}\right)$ is much larger than the spin-orbit contribution $\left(c_{2}\right)$ [2].

The mass shift due to strange quarks is given in the quark model by $\Delta M_{0 s}$. A comparison of this term with its large $N_{c}$ counterpart is given in Fig. 1, where we can see that the quark model predictions are always located within the error bars of the large $N_{c}$ results. In both approaches, one predicts a mass correction term due to $\mathrm{SU}(3)$-flavor breaking which decreases with $N$.
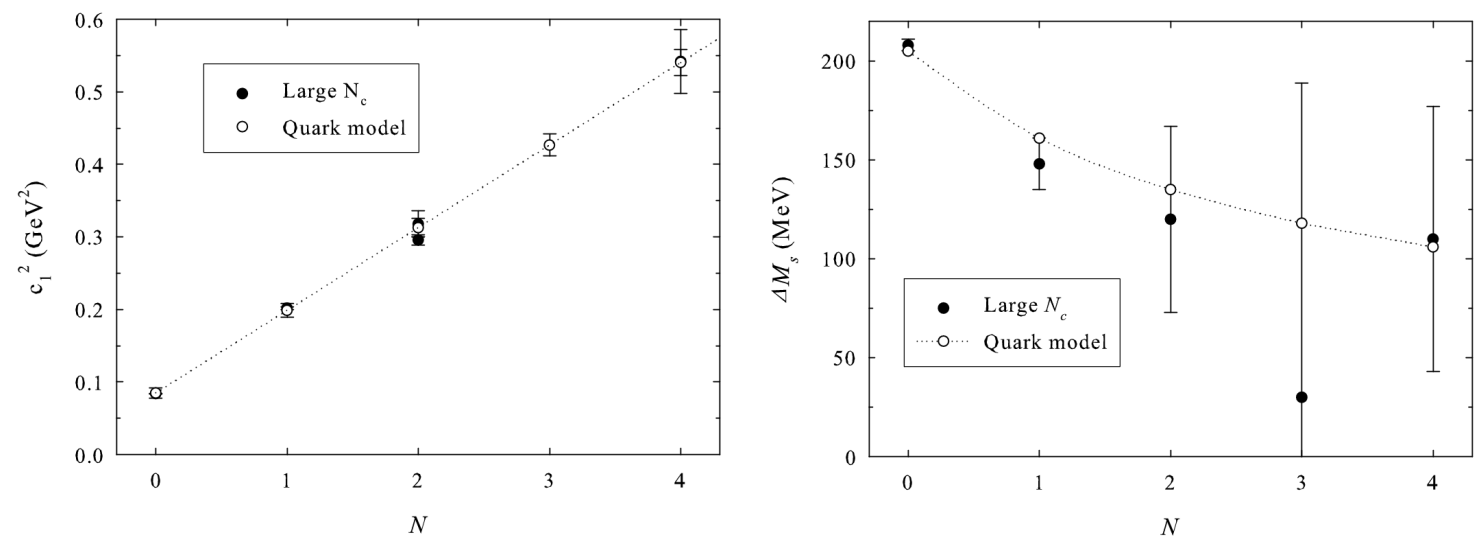

Figure 1: Plot of $c_{1}^{2}$ (left) and $\Delta M_{s}$ (right) versus the band number $N$. The values computed in the $1 / N_{c}$ expansion (full circles) from a fit to experimental data are compared with the quark model results with $\sigma=0.163 \mathrm{GeV}^{2}, \alpha_{0}=0.4, f=3.6$, and $m_{s}=0.240 \mathrm{GeV}$ (empty circles and dotted line are given to guide the eyes). The large $N_{c}$ data are nearly indistinguishable from the quark model prediction in the left plot. 


\subsection{Heavy baryons}

The heavy quark masses $m_{c}$ and $m_{b}$ can be independently fitted to the experimental data in both the quark model and the $1 / N_{c}$ frameworks [4]. In large $N_{c}$ QCD one obtains $m_{c}=1315 \mathrm{MeV}$ and $m_{b}=4642 \mathrm{MeV}$, while the quark model mass formula (2.3) is compatible with the experimental data provided that $m_{c}=1252 \mathrm{MeV}$ and $m_{b}=4612 \mathrm{MeV}$ (the other parameters have been fitted to light baryons). Both approaches lead to quark masses that differ by less than 5\%: They thus agree at the dominant order, where only $m_{Q}$ is present.

The other parameter involved in the large $N_{c}$ mass formula is $\Lambda$, which, in the ground state band, can be identified to the mass formula (2.3) as follows: $\Lambda=c_{0}=\left.\frac{1}{3} M_{1}\right|_{N=0}$. According to the large $N_{c}$ data one has $c_{0}=\Lambda \simeq 0.324 \mathrm{GeV}$ while the quark model gives $0.333 \mathrm{GeV}$, which means a very good agreement for the QCD scale $\Lambda$. The terms of order $1 / m_{Q}$ lead to the identity $c_{0}^{\prime}=\left.2 m_{Q} \Delta M_{Q}\right|_{N=0}$. The large $N_{c}$ parameter $\Lambda=0.324 \mathrm{GeV}$ gives $c_{0}^{\prime} \sim \Lambda^{2}=0.096 \mathrm{GeV}^{2}$ and the quark model gives $0.091 \mathrm{GeV}^{2}$, which is again a good agreement. Finally, the $\mathrm{SU}(3)$-flavor breaking term is proportional to $\varepsilon \Lambda_{\chi} \sim m_{s}$. One should have $\varepsilon \Lambda_{\chi}=\left.\frac{2}{\sqrt{3}} \Delta M_{1 s}\right|_{N=0}$ by definition of $\varepsilon \Lambda_{\chi}$; indeed the large $N_{c}$ value $\varepsilon \Lambda_{\chi}=0.206 \mathrm{GeV}$ and the quark model estimate $0.170 \mathrm{GeV}$ also compare satisfactorily. We point out that, except for $m_{c}$ and $m_{b}$, all the model parameters are determined from theoretical arguments combined with phenomenology, or are fitted on light baryon masses. The comparison of our results with the $1 / N_{c}$ expansion coefficients $c_{0}, c_{0}^{\prime}$ and $\varepsilon \Lambda_{\chi}$ are independent of the $m_{Q}$ values. So we can say that this analysis is parameter free.

An evaluation of the coefficients $c_{2}, c_{2}^{\prime}$, and $c_{2}^{\prime \prime}$ through a computation of the spin-dependent effects is out of the scope of the present spin-independent formalism. But at the dominant order, the ratio $c_{2}^{\prime \prime} / c_{2}$ should be similar to $\mu_{1}=356 \mathrm{MeV}$, which is roughly in agreement with Eq. (1.5) stating that $c_{2}^{\prime \prime} / c_{2} \sim \Lambda$.

\section{Conclusion}

In summary, we have established a connection between the quark model and the combined $1 / N_{c}, 1 / m_{Q}$ expansion both for light baryons and for heavy baryons containing a heavy quark. Our results bring reliable QCD-based support in favor of the constituent quark model assumptions and lead to a better insight into the coefficients $c_{i}$ encoding the QCD dynamics in the $1 / N_{c}$ mass operator.

\section{References}

[1] N. Matagne and F. Stancu, A new look at the [70,1-] baryon multiplet in the $1 / N_{c}$ expansion, Nucl. Phys. A 811 (2008) 291 [hep-ph/ 0610099 ].

[2] C. Semay, F. Buisseret, N. Matagne and F. Stancu, Baryonic mass formula in large $N_{c}$ QCD versus quark model, Phys. Rev. D 75 (2007) 096001 [hep-ph / 0702075 ].

[3] C. Semay, F. Buisseret and F. Stancu, Mass formula for strange baryons in large $N_{c}$ QCD versus quark model, Phys. Rev. D 76 (2007) 116005 [arXiv:0708.3291 [hep-ph]].

[4] C. Semay, F. Buisseret and F. Stancu, Charm and bottom baryon masses in the combined $1 / N_{c}$ and $1 / m_{Q}$ expansion versus quark model, Phys. Rev. D 78 (2008) 076003 [arXiv: 0808.3349 [hep-ph]]. 